\title{
Adición de lodos residuales en la elaboración de matrices de cerámicas
}

\author{
$\checkmark$ Natalia Fuentes Molina ${ }^{1}$ \\ SAMIR ALFONSO ISENIA LEÓN ${ }^{1}$ \\ JoSE GREgorio AsCEnCIO MENDOZA ${ }^{1}$
}

\section{Resumen}

En los procesos de alfarería, la incorporación de lodos en la producción de cerámicos es algo que se está estudiando actualmente, ya que esta puede constituirse como una solución ambientalmente para estos. Durante la presente investigación se evaluaron las características físicas mecánicas de ladrillos cerámicos con adiciones de lodos residuales de plantas de tratamiento de agua residual (PTRAR), determinando su uso como materiales de construcción, realizando esto en distintos momentos; Recolección y caracterización; Selección de porcentajes de adiciones y temperaturas y Determinación de las características físicas y mecánicas según la NTC-4017. Encontrándose compuestos similares entre las materias primas, arcillas, lodos seco y calcinado $\left(\mathrm{SiO}_{2}\right)$ 59; 56 y 57\%, $\left(\mathrm{Al}_{2} \mathrm{O}_{3}\right)$ 19; 11 y $12 \%$, y $\left(\mathrm{Fe}_{2} \mathrm{O}_{3}\right) 4 ; 6$ y $7 \%$. Las características de los ladrillos, mostraron mayor resistencia (29,8 MPa) y menores absorciones (15,53 y 19,49 \%) para ladrillos cocidos a temperaturas de $1000^{\circ} \mathrm{C}$. presenciando mejores resultados a esta temperatura.

Palabras claves: biosólidos, arcillas, material cerámico, resistencia, y absorción.

\section{Addition Of Residual Sludge In The Development of Ceramic Matrices}

\section{Abstract}

In pottery processes, the incorporation of sludge into the production of ceramics is something that is currently being studied, since this can be constituted as a solution environmentally for these. During the present investigation the mechanical physical characteristics of ceramic bricks with additions of residual sludge from wastewater treatment

1 Universidad de la Guajira. Riohacha, Colombia.

Autor de correspondencia: Fuentes Molina, N. (Natalia): Universidad de La Guajira. Km. 5 Vía Maicao - RiohachaLa Guajira. Teléfono: 3016590985.

Correo electrónico: nnfuentes@uniguajira.edu.co
Historia del artículo:

Artículo recibido: 16-II-2017 / Aprobado: 30-V-2019

Disponible online: 3 de julio de 2019

Discusión abierta hasta marzo de 2021 
plants (PTRAR) were evaluated, determining their use as construction materials, doing this at different times; Collection and characterization; Selection of percentages of additions and temperatures and Determination of physical and mechanical characteristics according to NTC-4017. Similar compounds are found among the raw materials, clays, dry and calcined sludge (SiO 2) 59; 56 and 57\%, (Al2O3) 19; 11 and 12\%, and (Fe 20 3) 4; 6 and 7\%. The characteristics of the bricks showed higher strength (29.8 MPa) and lower absorptions (15.53 and 19.49\%) for bricks cooked at temperatures of $1000{ }^{\circ} \mathrm{C}$. Seeing better results at this temperature.

Keywords: Biosolids, Clay, Ceramic, Resistence, and Absorption.

\section{A adição de lodo de esgoto na produção de matrizes cerâmicas}

\section{Resumo}

Nos processos de cerâmica, a incorporação de lodo na produção de cerâmica é algo que está a ser estudada, como esta pode ser estabelecido como uma solução para estes ambientalmente. Durante esta investigação das características físicas mecânicas de tijolos cerâmicos foram avaliados adições lodo de estações de tratamento de águas residuais (PTRAR), determinando o uso como materiais de construção, tornando-se em momentos diferentes; Coleta e caracterização; Seleção de adições percentagens e temperaturas e Determinação das características físicas e mecânicas de acordo com a NTC-4017. Encontrar compostos semelhantes entre matérias-primas, argilas e lamas seco calcinado (SiO2) 59; 56 e 57\%, (Al203) 19; 11 e 12\%, e (Fe203) 4; 6 e 7\%. As características dos tijolos, mostrou maior resistência (29,8 MPa) e absorções menores (15,53 e 19,49\%) para cozida a temperaturas de $1000{ }^{\circ} \mathrm{C}$ tijolos. testemunhando melhores resultados, a esta temperatura.

Palavras-chave: Biossólidos, Argila, Cerâmica, Resistência e Absorção.

\section{Introducción}

La rápida urbanización e industrialización a nivel mundial, está causando serios problemas ambientales, entre los cuales se encuentra el manejo, aprovechamiento y disposición final de altos volúmenes de lodos, generados en las plantas de tratamiento de aguas residuales a nivel mundial; convirtiéndose en uno de los desafíos más significativos de la actualidad (Shakir, et al., 2013; Lissy y Sreeja, 2014). Los problema en la mayoría de los países radican en los altos costos, la escases de vertederos, los riesgos para el medio ambiente y la salud humana de las comunidades aledañas, entre otros (Victoria, 2013; Pappu, et al., 2007).
Existe una fuerte demanda de reciclaje y reutilización ambientalmente segura con métodos efectivos de aprovechamiento de lodos residuales, debido a las dificultades para encontrar vertederos adecuados y dejar de lado disposiciones inadecuadas tradicionalmente empleadas (Sullivan, et al., 2010). Siendo según Andreola, et al., (2005) el sector de la construcción el más indicado para absorber estos residuos, debido a la gran cantidad de materia prima utilizada por el sector así como por el gran volumen de productos finales.

La industria cerámica en la búsqueda de alternativas compatibles con las necesidades de tratamiento de estos residuos, plantea su incorporación 
mediante el aprovechamiento del poder calorífico en sus procesos de combustión, la incorporación de residuos en la estructura de los materiales como parte de su propia matriz Sánchez y Carda (2002), Naga (2002), entre otros que buscan motivar el interés de los investigadores para convertirlo en una práctica común (Kadir y Rahim 2014; Cruz y Corpas 2011; Dhanapandian, et al., 2010).

Una de las grandes preocupaciones del sector de la construcción en este tipo de investigaciones, es que el uso de lodos residuales disminuye la calidad del ladrillo (Martínez, et al., 2012; Jaramillo y Agudelo, 2007) alterando algunas propiedades como resistencia a la compresión, absorción, durabilidad, aislamiento térmico y acústico, (Duggal, 2012; Kadir y Amira, 2012; Kadir y Rahim, 2014). Perspectiva que ha venido cambiando con las investigaciones (Babu y Ramana, 2013; Hegazy, et al., 2012; Victoria, 2013) que reportan los efectos positivos en las propiedades tales como menor peso, baja densidad, mejoras en las propiedades mecánicas con porcentajes de adición específicos, bajos consumo de energía, entre otros.

Adicionalmente, las tendencia actuales en los países europeos, buscan mediante procesos normativos, regular los coeficientes de transferencia de calor de los materiales de construcciones, con el fin de reducir el consumo final de energía; apoyase así la tendencia hacia la producción de ladrillos de aislamiento térmico (García, et al., 2010; Sutcu y Akkurt, 2009; Massó, 2010).

Partiendo de los principios de desarrollo sostenible, y apoyados en la tendencia a obtener materiales con consumo mínimo de energía, la producción de ladrillos incorporando residuos orgánicos en la matriz, viabiliza el aprovechamiento de los lodos procedentes de los sistemas de tratamiento de aguas residuales como lo han demostrado Duggal, (2012); Sutcu y Akkurt, (2009); Mekki, et al., (2008); Alonso, et al., 2010; Kim, et al., 2005; Andreola, et al., 2005 en estudios recientes.

La presente investigación, planteó un análisis térmico, para optimizar la calidad de las matrices ce- rámicas, con adiciones de lodos residuales; logrando mejoras en las propiedades físicas, químicas y biológicas de los ladrillos. Se evaluó el comportamiento químico de las reacciones de deshidroxilación de los lodos en las matrices cerámicas que tienen lugar a los $450-500{ }^{\circ} \mathrm{C}$ y las reacciones de descomposición de la materia orgánica a los $400-700{ }^{\circ} \mathrm{C}$, hasta la formación del material vítreo (Lissy y Sreeja, 2014); para tener control sobre los procesos de difusión, que faciliten la transformación de estas a dióxido de carbono y la cristalización de la sílice y demás componentes, a partir de los $900{ }^{\circ} \mathrm{C}$ para la vitrificación de las matrices cerámicas, necesarias para unir las partículas que le confieren durabilidad y resistencia al producto obtenido, evitando las tensiones térmicas excesivas en los deltas de temperatura.

A partir de lo anterior, se evaluó las características químicas y físicas de los lodos procedentes de las plantas de tratamiento de aguas residuales como materia prima para la industria cerámica y mejorar las propiedades físicas y mecánicas de los materiales obtenidos mediante el análisis térmico, acorde con las necesidades y tendencias actuales del sector de la construcción.

\section{Materiales y Métodos}

Para determinar el aprovechamiento del uso de lodos residuales como adición de remplazo por la arcilla en la elaboración de matrices de cerámicas que cumplan con las especificaciones de la normativa colombiana vigente en cuanto a los estándares de calidad exigidos para la industria cerámica es conveniente destacar tres momentos; $i$. Recolección y caracterización físico y química de los biosólidos y las arcillas ii. Selección de los porcentajes de las adiciones y las temperaturas de cocción de los ladrillos cerámicos y iii. Determinación de las características físicas y mecánicas de las matrices cerámicas a partir de los procedimientos y muestreos de ensayo como establece la NTC-4017 para mampostería estructural y no estructural en Colombia. 


\subsection{Recolección y caracterización fí-} sico y química de los biosólidos y las arcillas:

Las muestras de lodos provienen del subproducto generado en la planta de tratamiento de aguas residuales del municipio de Valledupar - Colombia, considerando que la producción de este residuo deriva problemas de manejo y disposición inadecuada, generando implicaciones ambientales en la zona debido a su composición química además de los altos volúmenes alcanzados. A los lodos se les realizo una combustión controlada a una temperatura de $550{ }^{\circ} \mathrm{C}$, en busca de analizar resultados obtenidos entre matrices cerámicas con adición lodos secosarcilla, matrices cerámicas lodos calcinados- arcilla y matrices cerámicas de solo arcilla. Por otra parte las arcillas provienen de origen natural de bancos de extracción ubicados en el sur del departamento de La Guajira- Colombia destinados para la elaboración de matrices cerámicas libres de impurezas y material contaminante.

Se le aplicaron a las distintas muestras un proceso de triturado y tamizado para obtener partículas de tamaños no mayor a $1,18 \mathrm{~mm}$ por consiguiente se garantizó una mezcla trabajable y adherente debido a la distribución granulométricas de las materias trabajadas. La caracterización de la arcilla y los lodos se realizó mediante el análisis de la composición mineralógica, la cual se detectó a través de la técnica espectrofotométrica de fluorescencia de rayos X, con el espectrofotómetro MagixPro PW-2440 Philips equipado con un tubo de rodio, con una potencia máxima de 4KW, con una sensibilidad de 200ppm en la detección de elementos pesados metálicos.

\subsection{Selección de los porcentajes de} las adiciones y las temperaturas de cocción de los ladrillos cerámicos

Se elaboraron 60 matrices cerámicas con adición de lodo residual a fin de establecer la mejor respuesta a las pruebas de resistencia a la compre- sión y a la absorción de agua (capilar ABSC, sumergida ABSS y ebullición ABSE), teniendo como referentes a los porcentajes de adición (lodos secos y lodos calcinados) y temperaturas empleadas en la cocción de las matrices cerámicas establecidos mediante el análisis de diferentes investigaciones entre las que se encuentran Victoria (2013); Hegazy et al.(2012); Mageed et al. (2011) se seleccionaron de esta forma porcentajes de 5,10, 15, 20 y $30 \%$ de adición de lodo residual, para ser cocidos a temperaturas de 800,900 y $1000{ }^{\circ} \mathrm{C}$.

$\mathrm{Al}$ mismo tiempo se elaboraron 6 matrices cerámicas en ausencia de lodos residuales, tomándolos como referencia de control para la comparación de los resultados frente a los que contienen lodo residual. La dosificación de los materiales en las mezclas mencionadas se realizó mediante relaciones de peso con el objetivo de conocer la mejor respuesta de adicción ante el remplazo de la arcilla, su homogenización de forma manual el agua fue suministrada mediante dos aplicaciones diferentes, la primera fue hidratando la mezcla durante 24 horas y la segunda fue manteniendo el aire saturado de agua, tratando de regularlo para producir mejor trabajabilidad, asentamiento y fluidez que facilite el trabajo en la obtención de las matrices cerámicas.

En toda la investigación se tomaron las especificaciones de diseño de la NTC-296, donde se establece las dimensiones modulares, holguras y tolerancias de las unidades de mampostería de arcilla cocida, para este caso fueron de $5 \mathrm{~cm}$ de ancho, 5 $\mathrm{cm}$ de alto, $10 \mathrm{~cm}$ de largo y un peso promedio de $0,36 \mathrm{~kg}$ de peso neto. Las matrices cerámicas fueron secadas a una temperatura ambiente de $40{ }^{\circ} \mathrm{C}$ durante 24 horas; acto seguido se realizó el proceso de cocción a $700{ }^{\circ} \mathrm{C}$ con el fin de eliminar rastros de materia orgánica durante una hora, para luego aumentar la cocción a diferentes temperaturas 800 , 900 y $1000{ }^{\circ} \mathrm{C}$ en una mufla, para luego hacer los análisis correspondientes de resistencia a compresión y absorción (ABSC, ABSS y ABSE). 


\subsection{Determinación de las caracterís-}

\section{ticas físicas y mecánicas de las matrices cerámicas}

A las matrices cerámicas se les examino su calidad de acuerdo a la normativa exigida en Colombia para verificar el cumplimiento de lo establecido en la NTC-4205 para la utilización de matrices cerámicas para mampostería estructural y no estructural donde está fijada una resistencia mínima de $20 \mathrm{MPa}$ y 14 Mpa respectivamente. Para la prueba de resistencia mecánica a la compresión y la absorción de agua: absorción capilar (ABSC), absorción de sumergida (ABSS) y absorción de ebullición (ABSE) se ensayaron las matrices cerámicas, teniendo en cuenta los procedimientos de muestreo y ensayo de los ladrillos cerámicos como establece la NTC-4017.

\section{Resultados}

\section{Caracterización de arcillas y}

\section{biosólidos}

Análisis granulométrico de arcillas y biosólidos: El análisis granulométrico mostró una clasificación según AASHTO de A2 para todas las muestras, es decir (arenas limosas y arcillosas), y según USCS de SM para arcilla - biosólido calcinado y SW para biosólido seco, es decir SM: limos arenosos y SW: arena bien gradada. En cuanto a las medidas de plasticidad por el método de límites de Atterberg indicaron un límite líquido de 58 y 30 \%, límite plástico de 29 y $25 \%$ y un índice de plasticidad de 29 y 5 $\%$ para arcilla y biosólido calcinado respetivamente.

Los índices de plasticidad de las materias primas, muestran mayor plasticidad en las arcillas (29 $\%)$, pudiendo clasificar estas como, arcillas de alta plasticidad según USCS en función de los límites de Atterberg, y como A-7-6 según AASHTO. Y el biosólido calcinado (5\%) como limos de baja plasticidad según USCS y A-4 según AASHTO.
Los mayores porcentaje para las tres muestras se encuentra en diámetros menores a 0,425 mm (malla $\mathrm{N}^{\circ} 40$ ), con porcentajes de 68,33; 61,16 y 67,49 \% para la arcilla, biosólido seco y biosólido calcinado respectivamente, siendo similares estos porcentajes para arcilla y biosólido calcinado. Los porcentajes menores a 0,075 mm (malla $\mathrm{N}^{\circ}$ 200), para las tres muestras fueron de 22,14; 2,19 y 16,94 \% para la arcilla, biosólido seco y biosólido calcinado respectivamente, siendo mayor el porcentaje de las arcillas. Con estos valores se puede asegurar que granulométricamente las arcillas están compuestas por materiales más finos, que los biosólidos; además, calcinar el biosólido reduce el tamaño de la partícula, debido a la volatilización del material orgánico.

Análisis de la composición química de arcillas y biosólidos: El análisis químico mediante fluorescencia de rayos $\mathrm{X}$, indica que las materias primas (arcilla, biosólidos seco y biosólidos calcinado), están mayoritariamente compuestos de óxido de silicio $\left(\mathrm{SiO}_{2}\right) 59 ; 56$ y 57\%, alúmina $\left(\mathrm{Al}_{2} \mathrm{O}_{3}\right) 19 ; 11$ y $12 \%$, óxido de hierro $\left(\mathrm{Fe}_{2} \mathrm{O}_{3}\right)$; 6 y $7 \%$, y otros componentes minoritarios tales como minerales de óxido de calcio ( $\mathrm{CaO})$, óxido de potasio $\left(\mathrm{K}_{2} \mathrm{O}\right)$, óxido de sodio $\left(\mathrm{Na}_{2} \mathrm{O}\right)$, óxido de magnesio (MgO).

Además se encontraron altos niveles de óxido de azufre $\left(\mathrm{SO}_{3}\right)$ presentes en los biosólidos seco $(7,25 \%)$ y calcinado $(8,18 \%)$ en comparación con las arcillas (0,14\%) evaluadas en la presente investigación, deja en evidencia, los posibles riesgos ambientales, que se pueden presentar con el uso de los ladrillos cerámicos elaborados con las adiciones que se plantearon en la investigación. Sin embargo, en las investigaciones de Durante y Lima, (2015); Cusido et al. (2003) menciona que las emisiones promedio de dióxido de azufre $\left(\mathrm{SO}_{2}\right)$ en los productos cerámicos elaborados con biosólidos son considerablemente bajas y están por debajo de los límites máximos permisibles establecidos en las normativas para calidad de aire. 
Figura 1. Curva Granulométrica de arcillas y biosólidos (seco y calcinado)
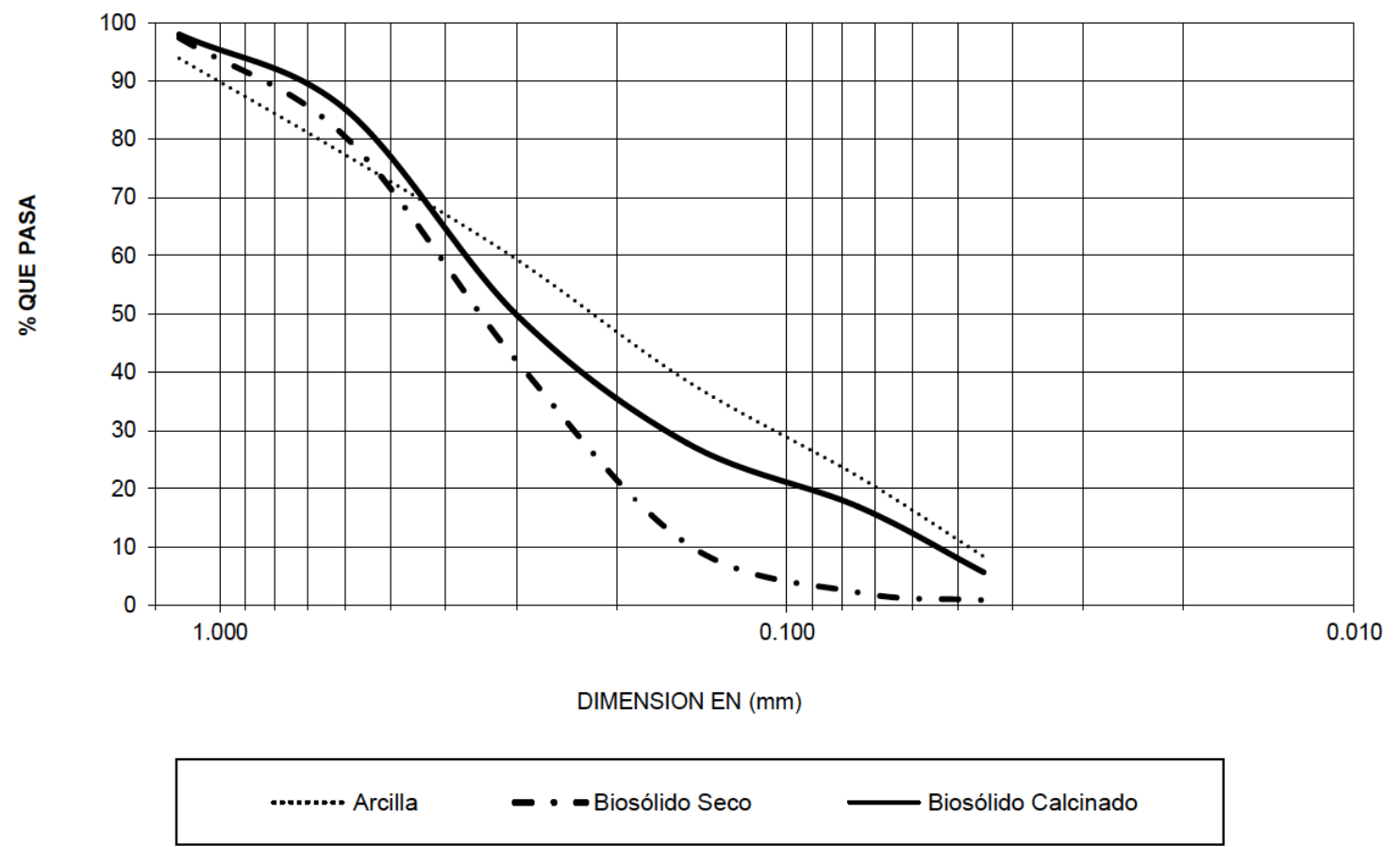

Figura 2. Análisis de la composición química de arcillas y biosólidos A. comparación química de materias primas (Arcilla AR, Biosólidos seco BS y Biosólido calcinado BC)

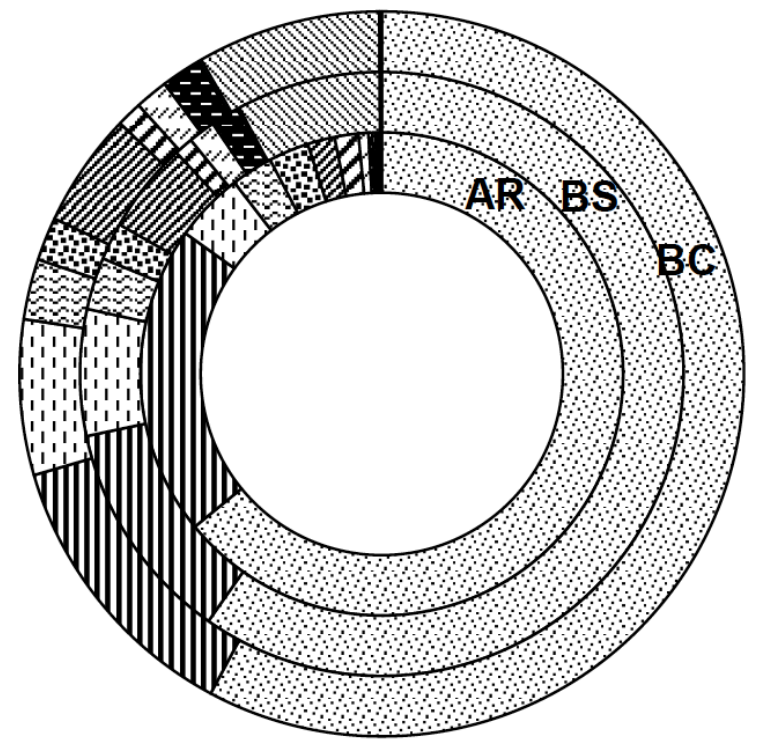

$\begin{array}{ll}\square \mathrm{SiO} 2 & \square \mathrm{Al} 2 \mathrm{O} 3 \\ \square \mathrm{Fe} 2 \mathrm{O} 3 & \square \mathrm{K} 2 \mathrm{O} \\ \square \mathrm{Na} 2 \mathrm{O} & \square \mathrm{CaO} \\ \square \mathrm{MgO} & \square \mathrm{TiO} 2 \\ \square \mathrm{P} 2 \mathrm{O} 5 & \square \mathrm{MnO} \\ \square \mathrm{Cl} & \square \mathrm{SO} 3\end{array}$

目Ba 
De lo anterior, se puede resaltar, que los compuestos mayoritarios de las materias primas, se encuentran distribuidos en porcentajes similares, en cada una de las muestras estudiadas, asemejándose la presencia de compuestos entre estos lo que lo cual benefician el aprovechamiento de los biosólidos, por su afinidad con las arcillas; facilitando la mezcla de los mismos en la elaboración de ladrillos cerámicos.

Otros análisis de las arcillas y biosólidos: Se encontraron contenido de humedad similar entre las arcillas (3,22 \%) y biosólidos seco (3,41\%); y contenido de materia orgánica es diferente entre estos, evidenciando la influencia que este puede tener en la elaboración de los ladrillos cerámicos, por la cual la presente investigación, planteo calcinar los biosólidos, como estrategia para reducir los contenido de materia orgánica (3,23\%); de forma tal que se aproxime a las características de las arcillas $(0,11 \%)$.

Se observa también correlación entre, las densidades y la granulometría; evidenciándose que las densidades más altas se presentan en las arcillas (2,40 mg/L), seguidas por el biosólido calcinado (2,39 mg/L) y el biosólido seco (1,63 mg/L).
Caracterización de ladrillos cerámicos con diferentes temperaturas de cocción

En los valores de contracción lineal de los ladrillos se observa una tendencia a aumentar en función de la temperatura, excluyendo los ladrillos cóccidos a temperaturas de $1000{ }^{\circ} \mathrm{C}$, los cuales mostraron resultados negativos, significando que algunos de estos ladrillos tuvieron un aumento lineal en una de sus dimensiones. El peso promedio de los ladrillos cerámicos, presento mayores valores en los ladrillos patrones $(365,83 \mathrm{~g})$, seguidos por los ladrillos con adiciones de biosólido calcinado $(360,32 \mathrm{~g})$ y biosólido seco (358,47 g), lo cual puede ser debido a las diferencias granulométricas de los materiales que influyen en la compactación de las mezclas.

\section{Determinación de las propiedades}

\section{físicas y mecánicas de los ladrillos cerámicos}

Análisis de resistencia a la compresión de los ladrillos cerámicos:

TABLA 1. RESISTENCIA A LA COMPRESIÓN (MPA), TASA INICIAL DE ABSORCIÓN O ABSORCIÓN CAPILAR ABC (G/ $\mathrm{CM}^{2} / \mathrm{MIN}$ ), ABSORCIÓN SUMERGIDA ABSS (\%) Y ABSORCIÓN POR EBULLICIÓN ABSE DE LADRILLOS CERÁMICOS CON BIOSÓLIDOS DE AGUAS RESIDUALES; PARA DIFERENTES TEMPERATURAS (HORNO, 800, $900 \mathrm{Y} 1000^{\circ} \mathrm{C}$ )

\begin{tabular}{|c|c|c|c|c|c|}
\hline \multicolumn{2}{|c|}{$\begin{array}{l}\text { Temperatura de coc- } \\
\text { ción }\left({ }^{\circ} \mathrm{C}\right)\end{array}$} & $\begin{array}{l}\text { Resistencia } \\
\text { (Mpa) }\end{array}$ & $\begin{array}{c}A B C \\
\left(\mathrm{~g} / \mathrm{cm}^{2} / \mathrm{min}\right)\end{array}$ & $\begin{array}{l}\text { ABSS } \\
(\%)\end{array}$ & $\begin{array}{l}\text { ABSE } \\
(\%)\end{array}$ \\
\hline \multicolumn{2}{|c|}{ TH } & $22,6 \pm 3,06$ & $0,40 \pm 0,02$ & $17,23 \pm 0,18$ & $20,30 \pm 0,20$ \\
\hline \multicolumn{2}{|c|}{800} & $26,2 \pm 2,94$ & $0,54 \pm 0,03$ & $17,34 \pm 0,14$ & $21,08 \pm 0,25$ \\
\hline \multicolumn{2}{|c|}{900} & $29,8 \pm 3,74$ & $0,54 \pm 0,04$ & $17,01 \pm 0,35$ & $20,94 \pm 0,27$ \\
\hline \multicolumn{2}{|c|}{1000} & $29,8 \pm 2,01$ & $0,63 \pm 0,05$ & $15,53 \pm 0,69$ & $19,49 \pm 0,69$ \\
\hline Kruskal & $\mathrm{K}-\mathrm{W}$ & 8,800 & 40,199 & 33,156 & 23,284 \\
\hline Wallis & $\mathrm{P}$ & 0,032 & 0,000 & 0,000 & 0,000 \\
\hline \multicolumn{2}{|c|}{ Tipo de tratamiento } & $\begin{array}{c}A B C \\
\left(\mathrm{~g} / \mathrm{cm}^{2} / \mathrm{min}\right)\end{array}$ & $\begin{array}{c}\text { ABSS } \\
(\%)\end{array}$ & \multicolumn{2}{|c|}{$\begin{array}{c}\text { ABSE } \\
(\%)\end{array}$} \\
\hline \multicolumn{2}{|c|}{ Biosólido seco } & $0,53 \pm 0,14$ & $16,51 \pm 1,35$ & \multicolumn{2}{|c|}{$20,39 \pm 1,74$} \\
\hline \multicolumn{2}{|c|}{ Biosólido calcinado } & $0,53 \pm 0,15$ & $17,02 \pm 1,26$ & \multicolumn{2}{|c|}{$20,53 \pm 1,10$} \\
\hline Kruskal & K-W & 0,000 & 4,084 & \multicolumn{2}{|c|}{0,017} \\
\hline Wallis & $P$ & 0,994 & 0,043 & \multicolumn{2}{|c|}{0,898} \\
\hline
\end{tabular}


Figura 3. Absorciones de ladrillos cerámicos con biosólidos de aguas residuales en función de las temperaturas $\left(800^{\circ} \mathrm{C}\right.$, $900^{\circ} \mathrm{C}, 1000^{\circ} \mathrm{C}$ y horno), A) Absorción capilar ABSC, B) Absorción sumergida (ABSS) y C) Absorción por ebullición $\mathrm{ABSE}$, en función del tratamiento del biosólido D) ABSC, E) ABSS, F) ABSE
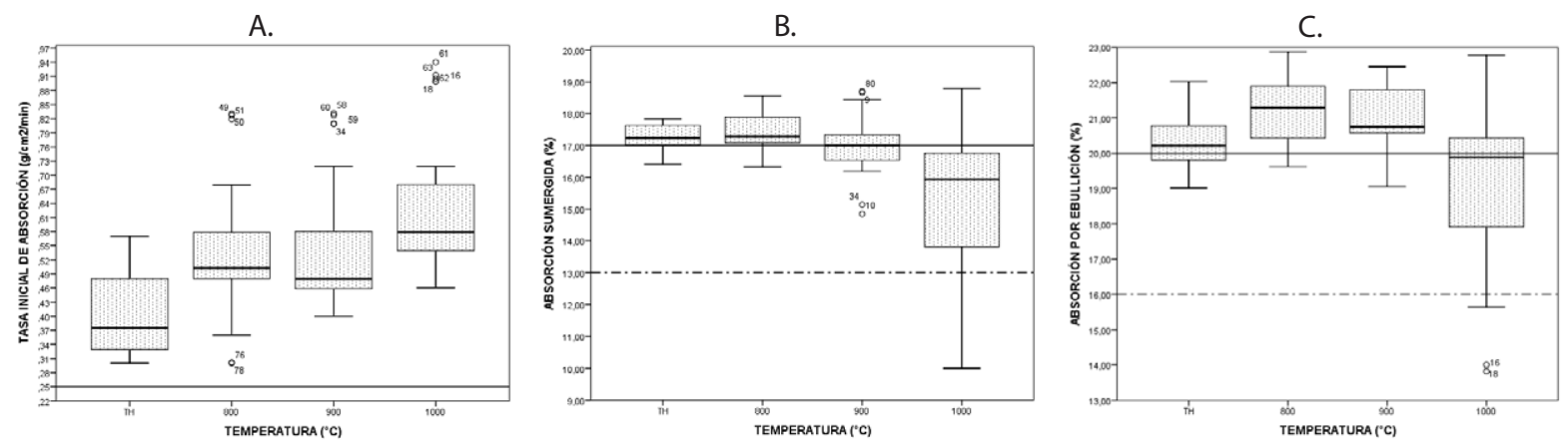

D.

E.
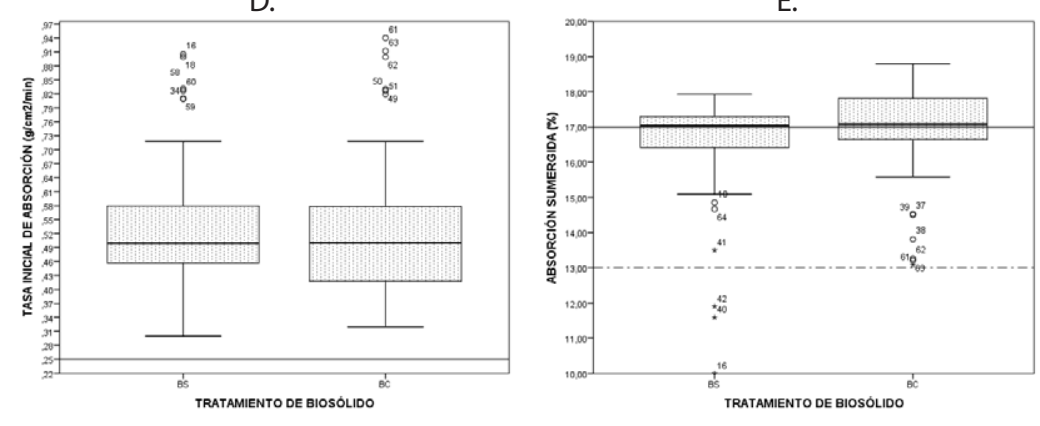

F.

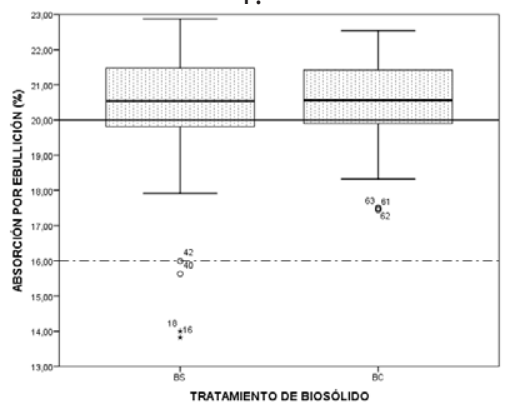

En función de la temperatura: Las relaciones entre la resistencia a la compresión de los ladrillos cerámicos y las diferentes temperatura evaluadas (Horno, $800^{\circ} \mathrm{C}, 900{ }^{\circ} \mathrm{C}$ y $1000^{\circ} \mathrm{C}$ ), presentaron diferencias significativas (K-W: 8,800; $\mathrm{P}<0,032 ; \mathrm{N}$ : 120); donde se evidencia que los valores promedios más altos registrados fueron de 36,6; 39,7; 40,7 y 48,5 Mpa respectivamente. Encontrando que la mayor resistencia de la compresión fue 51,0 MPa para una temperatura de $1000{ }^{\circ} \mathrm{C}$ de cocción, y la menor resistencia a compresión fue de 10,0 MPa en donde su proceso de cocción se manejó las temperaturas internas de hornos artesanales.

Los ladrillos patrón (100\% de arcilla) presentaron un comportamiento similar; con resistencias de 26,$3 ; 22,2 ; 34,0$ y $36,6 \mathrm{MPa}$ para las temperaturas de horno, 800; 900 ; y $1000{ }^{\circ} \mathrm{C}$ respectivamente. Evidenciando la existencia de una relación directamente proporcional entre la temperatura y la resisten- cia; por consiguiente todos los ladrillos cerámicos cocidos a diferentes temperaturas cumplen con la mampostería no estructural, y ciertos casos de estos ladrillos cumplen para mampostería estructural establecidos por la norma NTC-4205.

Análisis de absorciones de agua de los ladrillos cerámicos:

En función de la temperatura: La relación entre la tasa inicial de absorción o absorción capilar (ABSC) con respecto a la temperatura, es directamente proporcional, con diferencias significativas (K-W: 40,199 P<0,000, N: 120); en donde se evidencia que los valores promedios máximos registrados fueron de 0,$40 ; 0,54 ; 0,52$ y $0,62 \mathrm{~g} / \mathrm{cm}^{2} / \mathrm{min}$ para las temperaturas de Horno, 800,900 y $1000{ }^{\circ} \mathrm{C}$ respectivamente, todos estos incumplen la norma NTC4205, porque sobrepasan el límite máximo $(0,25 \mathrm{~g} /$ $\mathrm{cm}^{2} / \mathrm{min}$ ) establecido. 
Aunque los ladrillos cerámicos no cumplen con límite referenciado en la norma, esto no demerita la calidad del ladrillo obtenido, lo que se debe tener en cuenta para estos casos es "vigilar el cumplimiento de los requisitos de absorción y resistencia, y diseñar los morteros de pega y tiempos de pre-humedecimiento apropiados". Se debe resaltar, además que los valores promedios $\left(0,47 \mathrm{~g} / \mathrm{cm}^{2} / \mathrm{min}\right)$ en los ladrillos patrón también superan el límite referenciado en la norma NTC-4205 para mampostería en general.

La absorción sumergida (ABSS) y la temperatura presentaron una relación inversamente proporcional, con diferencias significativas $(\mathrm{K}-\mathrm{W}$ : $33,156 \mathrm{P}<0,000, \mathrm{~N}: 120$ ); en donde se evidencia que los valores promedios registrados fueron de 17,23; 17,$34 ; 17,01$ y $15,53 \%$ para las temperaturas Horno; 800,900 y $1000{ }^{\circ} \mathrm{C}$ respectivamente; en los cuales, solo cumplen la normativa NTC-4205, los ladrillos elaborados con temperaturas de $1000{ }^{\circ} \mathrm{C}$ para mampostería no estructural. Se evidenció además, que el promedio de los ladrillos patrón $(16,01 \%$ ) se encuentra por debajo de los límites máximo, establecido por la norma NTC-4205, para mampostería no estructural (17\%) y por encima de los límites máximos para mampostería estructural (13\%).

La relaciones entre absorción por ebullición (ABSE) y temperatura son inversamente proporcionales, registrando diferencias significativas (K-W: 23,284 $\mathrm{P}<0,000, \mathrm{~N}: 120)$ entre estas; en donde se evidencia que los valores promedios registrados fueron de 20,30; 21,08; 20,94 y 19,49\% para las temperaturas Horno, 800; 900 y $1000{ }^{\circ} \mathrm{C}$ respectivamente, mostrándose que en algunos casos se cumple el límite máximo establecido en la norma NTC-4205, para mampostería no estructural (20 \%) y muy pocos casos para mampostería estructural (16\%). En cuanto a los ladrillos patrones $(19,56 \%)$ se evidencia su cumplimiento para mampostería no estructural.

En función del tratamiento del biosólido: La tasa inicial de absorción (ABSC) y el tratamiento del biosólido no presentan diferencias significativas (K$\mathrm{W}: 0,000 \mathrm{P}>0,994, \mathrm{~N}: 120$ ); con valores promedios de $0,53 \mathrm{~g} / \mathrm{cm}^{2} / \mathrm{min}$ para biosólido seco, $0,53 \mathrm{~g} / \mathrm{cm}^{2} /$ min para biosólido calcinado y $0,47 \mathrm{~g} / \mathrm{cm}^{2} / \mathrm{min}$ para patrón, evidenciándose que todos estos incumplen la norma, porque sobrepasan el límite máximo $(0,25$ $\mathrm{g} / \mathrm{cm}^{2} / \mathrm{min}$ ) establecido en la NTC-4205.

La relación entre absorción sumergida (ABSS) y el tratamiento del biosólido presentan diferencias significativas (K-W: 4,084 $\mathrm{P}<0,043, \mathrm{~N}: 120$ ); con valores promedios de $16,51 \%$ para biosólido seco, 17,02 \% para biosólido calcinado y 16,01 \% para patrón, evidenciándose que estos cumplen para algunos casos (biosólido seco y patrón) el límite máximo establecido en la NTC-4205 para mampostería no estructural (17 \%) y en ningún caso para mampostería estructural (13\%).

En cuanto a la absorción por Ebullición (ABSE) y el tratamiento del biosólido no existen diferencias significativas (K-W:0,017, P>0,898, N: 120); con valores promedios de 20,39 \% para biosólido seco, 20,53 \% para biosólido calcinado y $19,56 \%$ para patrón, presentado que los ladrillos con adiciones no cumplen con la normativa para mampostería estructural y no estructural 16 y $20 \%$ respectivamente.

Finalmente se puede observar que los ladrillos elaborados con biosólido calcinado, lograron absorber mayor cantidad de agua, que los ladrillos patrón y los elaborados con biosólido seco, lo cual es debido posiblemente al bajo contenido de humedad. Sin embargo se esperaba que por la ausencia de plasticidad en el biosólido seco, se presentaran en este, las mayores absorciones de agua.

\section{Discusión}

Los resultados de la clasificación AASTHO para todas las muestras presentaron similitud entre los resultados presentados por Victoria, (2013), quien reporto una clasificación de arena de arcilla fina y suelo de arcilla para el lodo y la arcilla respectivamente.

Los resultados del análisis químico por fluorescencia de rayos $\mathrm{X}$, mostraron una afinidad entre los lodos y la arcilla debido a la similitud en los porcentajes de los componentes mayoritarios, estos resultados se asemejan a los resultados encontrados 
por Sandeep et al., (2014) quien encontró el mayor porcentaje de los componentes de lodos de PTAR, de $57,4 \%$ para $\mathrm{SiO}_{2}$, y $7,8 \%$ para $\mathrm{Al}_{2} \mathrm{O}_{3}$, a diferencia de los resultados para lodos de PTAP, encontrados por Ribeiro et al., (2002) y Mageed et al., (2011) quienes reportaron porcentajes más altos $\mathrm{de}_{2} \mathrm{Al}_{2} \mathrm{O}_{3} \mathrm{y}$ menores de $\mathrm{SiO}_{2}$.

La influencia de la temperatura en la elaboración de ladrillos cerámicos mediante mezclas lodos-arcilla es directamente proporcional con la resistencia a la compresión, al igual que los resultados presentados en las investigaciones de Mozo et al., (2015), Hegazy et al., (2012) y Acuña, (2008). Los valores promedio para los ladrillos cocidos a $1000{ }^{\circ} \mathrm{C}$ alcanzaron resistencias de hasta $48,7 \mathrm{MPa}$, valores similar al reportado por Mozo et al. (2015), quien evidenció resistencias de 50,26 $\mathrm{MPa}$ a una temperatura de $1050{ }^{\circ} \mathrm{C}$, e inferior al reportado por Acuña, (2008) quien evidenció resistencia de 22,72 MPa a una temperatura de $900{ }^{\circ} \mathrm{C}$; estos promedios de resistencias a la compresión se encontraron en las máximas temperaturas trabajadas por los autores.

Esta relación directamente proporcional entre la resistencia a la comprensión y la temperatura es debido al alto contenido de sílice $\left(\mathrm{SiO}_{2}\right)$ de las mezclas, el cual es un componente común de los suelos de fabricación de ladrillos, este cambia a su forma cristalina a temperaturas superiores a $573{ }^{\circ} \mathrm{C}$. La formación de vidrio, es necesaria para unir las partículas y hacer que el producto sea fuerte y duradero, comienza a aproximadamente $900{ }^{\circ} \mathrm{C}$, como indica Lissy y Sreeja (2014).

Por otra parte, la tasa inicial de absorción (TIA) se correlaciono en forma significativa con respecto a la temperatura con resultados promedio de $0,30 \mathrm{~g} / \mathrm{cm}^{2} / \mathrm{min}$ y $0,94 \mathrm{~g} / \mathrm{cm}^{2} / \mathrm{min}$ para una temperaturas de $800{ }^{\circ} \mathrm{C}$ y $1000{ }^{\circ} \mathrm{C}$ respectivamente, lo cual se puede comparar con los autores (Mozo et al. 2015), que registra absorciones de $0.28 \mathrm{~g} / \mathrm{cm}^{2} / \mathrm{min}$ y $0.32 \mathrm{~g} / \mathrm{cm}^{2} / \mathrm{min}$ de TIA en rangos de temperaturas de $950{ }^{\circ} \mathrm{C}$ y $1050{ }^{\circ} \mathrm{C}$ respectivamente, confirmando que existe una relación directamente proporcional entre estas dos variables.
Las absorciones sumergida (ABSS), presentaron una relación inversamente proporcional con respecto a la temperatura, resultaros similares a los presentados por Victoria, (2013) y Hegazy et al., (2012), quienes reportaron menores porcentajes de absorción en las temperaturas de $1050^{\circ} \mathrm{C}$ y $1200^{\circ} \mathrm{C}$ respectivamente, las cuales fueron las mayores temperaturas trabajadas por estos autores. Se puede afirmar que existe una correlación significativa entre la absorción sumergida y la temperatura, similar con (Mozo et al. 2015) que presentaron iguales variaciones donde a mayor temperatura menor ABSS, inversamente cuando se trabaja a menor temperatura mayor será la ABSS.

Además se encontró una similitud en la tendencia en las absorciones por ebullición (ABSE) y sumergida (ABSS), resultados similares a los presentados por Prashant $y$ Arun (2013), quienes encontraron que, en el caso del ensayo de agua hirviendo, el patrón de absorción de agua es el mismo que el del agua fría. Sin embargo, en este caso, el \% de absorción de agua es más en comparación con la prueba de agua fría.

\section{Conclusión}

Los resultados de esta investigación fortalecen la estrategia del aprovechamiento de los lodos generados en las plantas de tratamiento de aguas residuales como adicción de remplazo de arcilla para la elaboración de matrices cerámicas, los cuales evidencian óptimos resultados en las pruebas físico-mecánicas realizadas a los diferentes ladrillos, igualmente representa importantes beneficios ambientalmente, ya que los contaminantes de origen orgánico (nutrientes, materia orgánica y agentes patógenos) resultan inactivos al momento de la cocción de los materiales cerámicos, similarmente compuestos metálicos pesados presente en los lodo residual quedan encapsulados, evitando así, su mal manejo e inadecuada disposición final; planteando con esto, un aprovechamiento más seguro, al mismo tiempo que reduce el consumo de arcilla como materia principal en la elaboración de matrices cerámicas. 
La caracterización de la arcilla y los lodos demuestra mayor afinidad y similitud en los componentes de las muestras para los lodos de PTAR debido a la similitud en el contenido de $\mathrm{SiO}_{2}$ de estos, a diferencia de los porcentajes presente en los lodos de PTAP quienes presentan mayor porcentaje de compuestos aluminosos, siendo los lodos de PTAR los más adecuados para ser utilizados como sustituto de la arcilla en la fabricación de materiales cerámicos.

Las resistencias a la compresión analizadas en los ladrillos cerámicos muestran una relación directamente proporcional, al aumentar la temperatura de cocción la resistencia a la compresión de los matrices cerámicos aumenta similarmente, esto se logra evidenciar en temperaturas trabajadas de $1000{ }^{\circ} \mathrm{C}$ alcanzando una resistencia de $51,0 \mathrm{Mpa}$, así mismo para temperaturas trabajadas en hornos artesanales $\left(750{ }^{\circ} \mathrm{C}\right)$ la resistencia a la comprensión alcanzo $10 \mathrm{Mpa}$. Es de resaltar que los ladrillos cerámicos con adición de lodos procedente de las PTAR, presentan resistencias superiores a los ladrillos patrones en todas las pruebas realizadas, indicando que al agregar biosólidos a la mezcla de arcilla para la elaboración de ladillos cerámicos se incrementa la propiedad de resistencia en estos.

Las pruebas de absorción de agua en los ladrillos cocidos a $\left(800,900^{\circ} \mathrm{C}\right)$ no lograron cumplir con la normativa vigente, sobrepasando los límites permitidos, por el contrario solo lograron cumplir en gran parte los ladrillos cocidos a un temperatura de $1000{ }^{\circ} \mathrm{C}$, asignándolos para mampostería no estructural para este caso en específico.

Por otra parte, se demuestra que el tratamiento sometido a los lodos (seco - calcinado) no representa diferencias significativas en los resultados de las pruebas mecánicas valoradas, no obstante se lograron obtener mayores resultados en los ladrillos cerámicos con adición de lodos calcinado que los ladrillos cerámicos con adición del lodos seco en las pruebas físico-mecánicas realizadas.

También se puedo verificar que la introducción de los lodos no interfiere en la conformación del material, durante el proceso de moldeo y pren- sado, sin embargo después del proceso térmico, se obtiene un material con mayor grado de contracción, mayor porosidad y por ende mayor capacidad de absorber agua, lo cual permite inferir que estos materiales presentaran una tendencia al desgaste y a la hidratación, por lo cual se sugiere ubicarlos en lugares preferenciales en comparación con los ladrillos comerciales elaborados de solo arcillas.

\section{Referencias}

ACUÑA, N. 2008. Valorización de lodos residuales de la producción de sulfato de manganeso en Quintal S.A. Prospectiva 6(2): 31- 37.

AESLINA, A.K; AHMAD, A.R. 2014. An overview of sludge utilization into fired clay brick, World Academy Of Science, Engineering And Technology, International Journal Of Environmental, Chemical, Ecological, Geological And Geophysical Engineering Vol:8, No:8.

ALONSO, A; ÁlVAREZ, J; BUENO, S. 2010. Posibilidades de uso de las materias primas cerámicas del área de Bailén (Jaén). Modificación de sus propiedades mediante la utilización de residuos industriales, Libro de Ponencias I Congreso Nacional de Minerales Industriales, Fueyo Editores Instituto Geológico y Minero de España, pp. 175-179.

ANDREOLA, F; BARBIERI, L; LANCELLOTTI, I; POZZI, P. 2005. Reciclado de residuos industriales en la fabricación de ladrillos de construcción, 1st part, Materiales de Construcción 55 (280) (2005) 5-16.

BABU, G; RAMANA, N. 2013. Durability of Bricks Cast with Industrial Sludge, IOSR Journal of Machanical and Civil Engineering (IOSR-JMCE), vol. 6(4), pp. 43-46.

CRUZ, N; CORPAS, F. A. 2011. The use of different forms of waste in the manufacture of ceramic bricks, Applied Clay Science, vol. 52, pp. 270-276, 2011.

CUSIDO, J.A.; CREMADES, L.V.; GONZÁLEZ, M. 2003. Gaseous emissions from ceramics manufactured with urban sewage sludge during firing processes. Waste Management, (23):273-280.

DHANAPANDIAN, S; SHANTHI, M; MANOHARAN, C; RAMKUMAR, T; DEIVEEGAN, A. 2010. Investigation of Granite Waste Incorporated Clay Brick as A Building Material, International Journal of Recent Scientific Research, vol 4, pp. 107-113. 
DUGGAL, S.K. 2012. Building Materials. New Age International Publishers, New Delhi. pp. 8- 33, 234238,315-319.

DURANTE, M.P; LIMA A.D. 2015. Use of Sewage Sludge as Raw Material in the Manufacture of Roofs. Materials and Environmental Sciences CMES. Págs.31-33.

GARCÍA, J; ORTS, M.J; SABURIT, A; SILVA, G. 2010. Thermal conductivity of traditional ceramics. Part I: influence of bulk density and firing temperature, Ceramics International 36 1951-1959.

HEGAZY, B.E; FOUAD, H.A; HASSANAIN, A.M. 2012. Australian Journal of Basic and Applied Sciences, 6(3): 453-461, ISSN 1991-8178.

ICONTEC. Instituto Colombiano de Normas Técnicas y Certificación. Norma técnica Colombiana - NTC 4017. 2005. Métodos para muestreo y ensayos de unidades de mampostería y otros productos de arcilla.

ICONTEC. Instituto Colombiano de Normas Técnicas y Certificación. Norma técnica Colombiana - NTC 296. 2000. Dimensiones modulares de unidades de mampostería de arcilla cocida. Ladrillos y bloques cerámicos.

ICONTEC. Instituto Colombiano de Normas Técnicas y Certificación. Norma técnica Colombiana - NTC 4205. 2000. Unidades de mampostería de arcilla cocida. Ladrillos y bloques cerámicos.

JARAMILLO, L.Y; AGUDELO, S.C. 2007. Desechos con potencial industrial, Colombia Ciencia y Tecnología 23 (3).

KADIR, A; AMIRA, N. 2012. An overview of wastes recycling in fired clay bricks, International Journal of Integrated Engineering, vol 4(2), pp. 53-69.

KIM, Y; KIM, J.H; LEE, K.G; KANG, S.G. 2005. Recycling of dustwastes as lightweight aggregates, Journal of Ceramic Processing Research 6 (2) 91-94.

LISSY, P.N; SREEJA, M.S. 2014. Utilization of sludge in manufacturing Energy Efficient Bricks. IOSR Journal of Mechanical and Civil Engineering (IOSR-JMCE) eISSN: 2278-1684, p-ISSN: 2320-334X, Volume 11, Issue 4 Ver. III Jul- Aug., PP 70-73.

MAGEED, A.A; RIZK, SH.A; ABU-ALI, M.H. 2011. Journal of Engineering Sciences, Assiut University, Vol. 39, No 1, pp. 195-206, January.

MARTÍNEZ, C; COTES, T; CORPAS, F.A. 2012. Recovering wastes from the paper industry: Development of ceramic materials, Fuel Processing Technology 103, 117-124.

MASSÓ, Y. 2010. Eficiencia energética y sostenibilidad en los edificios, 253, Rev. AENOR, pp. 20-23.

MEKKI, H; ANDERSON, M; BENZINA, M; AMMAR, E. 2008. Valorization of olive mill wastewater by its incorporation in building bricks, Journal of Hazardous Materials 158 308-315.

MOZO, W; GÓMEZ, A; CAMARGO, G. 2015. Efecto de la adición de biosólidos (secos) a una pasta cerámica sobre la resistencia mecánica de los ladrillos. Revista Ingenierías Universidad de Medellín. ISSN 1692 - 3324. Medellín. Colombia. 14(27):61- 78.

NAGA, S.M. 2002. A. El-Maghraby, Industrial wastes as raw materials for tile making, Key Engineering Materials 206 1787-1790.

PAPPU, A; SAXENA, M; ASOLEKAR, S.R. 2007. Solid waste generation in India and their recycling potential building materials. Journal of Building and Environmental 42 (6): 2311-2324.

PRASHANT, G.S; ARUN, K.D. 2013. Technical Properties of Pond Ash - Clay Fired Bricks - An Experimental Study. American Journal of Engineering Research (AJER), e-ISSN: 2320-0847 p-ISSN: 2320-0936, Volume-02, Issue-09, pp-110-117.

RIBEIRO, M.J; TULYAGANOV, D.U; FERREIRA, J.M; LABRINCHA, J.A. 2002. Recycling of Al-rich industrial sludge in refractory ceramic pressed bodies, Ceramics International 28 319-326.

SÁNCHEZ, L; CARDA, J.B. 2002. Materiales residuales, Materias primas y aditivos cerámicos, Faenza Editrice Iberica, S.L., Castellón, pp. 159-160.

SANDEEP, Y; SUYASH, A; SHIVAM, G; RISHABH, K.T. 2014. Incorporation of STP Sludge and Fly ash in Brick Manufacturing: An attempt to save the Environment, International Journal of Advancements in Research \& Technology, Volume 3, Issue 5, May, ISSN 2278-7763.

SHAKIR, A; NAGANATHAN, S; MUSTAPHA, K. 2013. Properties of bricks made using fly ash, quarry dust and billet scale. Construction and Building Materials. Págs.131-138.

SULLIVAN, C; TYRER, M; CHEESEMAN, C.R; GRAHAM, N.J.D. 2010. Disposal of Water Treatment Wastes Containing Arsenic - A Review, Science of the Total Environment, 408: 1770-1778. 
SUTCU, M; AKKURT, S. 2009. The use of recycled paper processing residues in making porous brickwith reduced thermal conductivity, Ceramics International 35 2625-2631.

VICTORIA, A.N. 2013. Characterisation and Performance Evaluation of Water Works Sludge as Brick Material, International Journal of Engineering and Applied Science, vol. 3(3), pp. 69-79. 